\title{
Analysis of Polymer-Biomacromolecule Composites in the Solid-State via Energy Dispersive Spectroscopy-Scanning Electron Microscopy
}

\author{
Parker W. Lee ${ }^{1}$, Nanthawan Avishai ${ }^{2}$ and Jonathan K. Pokorski ${ }^{1}$ \\ 1. Department of Macromolecular Science and Engineering, Case Western Reserve University, Cleveland, \\ $\mathrm{OH}$, USA. \\ 2. Swagelok Center for Surface Analysis of Materials, Case Western Reserve University, Cleveland, OH, \\ USA.
}

The analysis and mapping of the dispersion of biomacromolecules within solid-state polymeric materials prepared via melt-processing is important in understanding the aggregation behavior in response to different loading levels, additives, and biochemical properties of the biomacromolecules. The presence of biomacromolecules can impart new properties to the material, such as flame retardant properties, or therapeutic value through controlled release from the polymer matrix [1]. A major issue in the meltprocessing of biomacromolecules is the high temperatures necessary, typically within the range of $95-$ $200{ }^{\circ} \mathrm{C}$. These elevated temperatures can cause the biomacromolecules to aggregate and denature within the polymer melt and remain once cooled [2]. The aggregation can result in loss of the desired activity of the biomacromolecule once released, irregular release profiles, or segregation of the biomacromolecule within the matrix resulting in diminished overall composite mechanical properties.

The particular biomacromolecules of interest for this study were deoxyribonucleic acid (DNA) and viruslike particles (VLPs) as additives. The DNA was melt-processed with low-density polyethylene (LDPE) to impart flame retardant properties and the VLPs were melt-processed with poly(lactic-co-glycolic acid) (PLGA) to create sustained release depot systems. Backscattered electron (BSE) imaging is not sufficient to distinguish the difference between polymer and biomacromolecule because both largely consist of carbon. Another limitation with BSE imaging is the mass contrast can be easily overwhelmed by topographic contrast. However, energy dispersive spectroscopy (EDS) in scanning electron microscopy (SEM) is a valuable tool for biomacromolecule detection. Using the distinct chemical signatures in biomacromolecules, such as nitrogen, sulfur, and phosphorus, biomacromolecule dispersion can be successfully detected [3]. Here we report the impact of sample preparation and collection conditions on EDS mapping for DNA and VLP elemental signatures.

The effects of freeze-fracturing versus cryo-microtoming surface preparation on the apparent dispersion of DNA within the LDPE matrix was investigated at two different concentrations of DNA. Cryomicrotoming the surface enhanced the ability to correctly discern and co-localize the elemental signals from DNA within the LDPE matrix. The interplay between image quality and signal intensity for VLPs embedded within PLGA was also explored in this study. PLGA deteriorates rapidly under high voltages, however higher voltages are necessary to yield signal from the relatively low-loading levels of VLPs and the elemental percent of VLP that is a distinguishable elemental signal. The effective voltage necessary for mapping the signal, while allowing for the preservation of the SEM image and visualization of the distribution of VLPs within the PLGA matrix was determined. These studies demonstrate the power of EDS-SEM for mapping biomacromolecules in solid-state polymer matrices and highlights considerations in sample preparation and signal collection to yield optimal data. 


\section{References:}

[1] S. A. Isarov, P. W. Lee, J. H. Towslee, K. M. Hoffman, R. D. Davis, J. M. Maia and J. K. Pokorski, Polymer. 97 (2016), 504-514.

[2] Z. Ghalanbor, M. Körber and R. Bodmeier, Int. J. Pharm. 438 (2012), 302-306.

[3] E. Wyroba, S. Suski, K. Miller and R. Bartosiewicz, Cell. Mol. Biol. Lett. 20 (2015), 488-509.

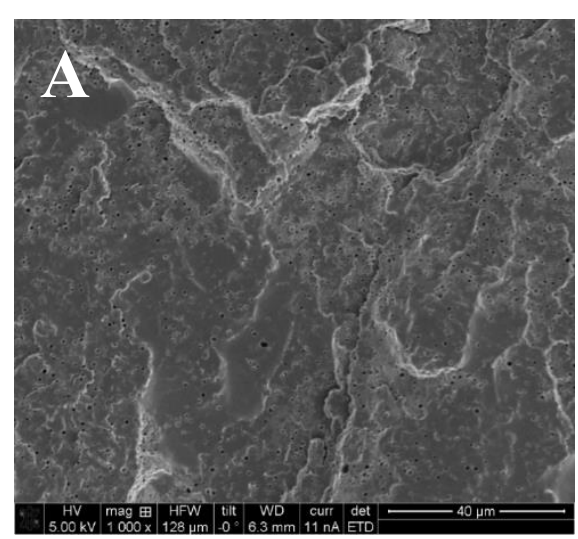

$\mathrm{N} \mathrm{K}$ series

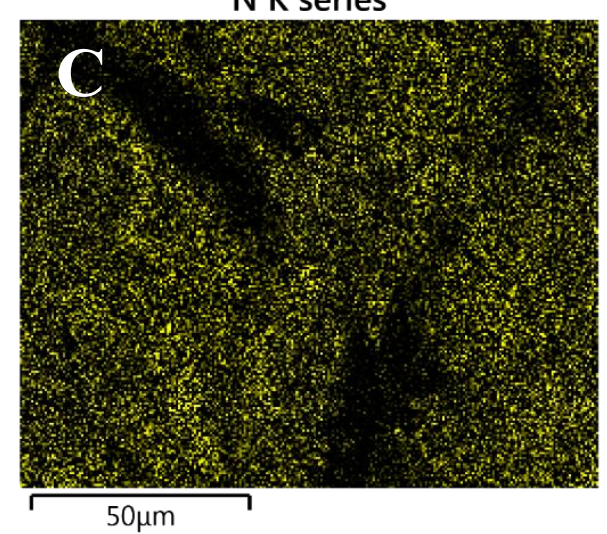

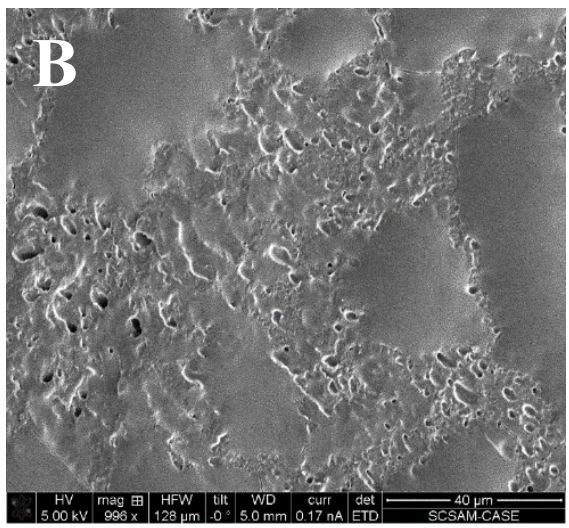

N K $\alpha 12$

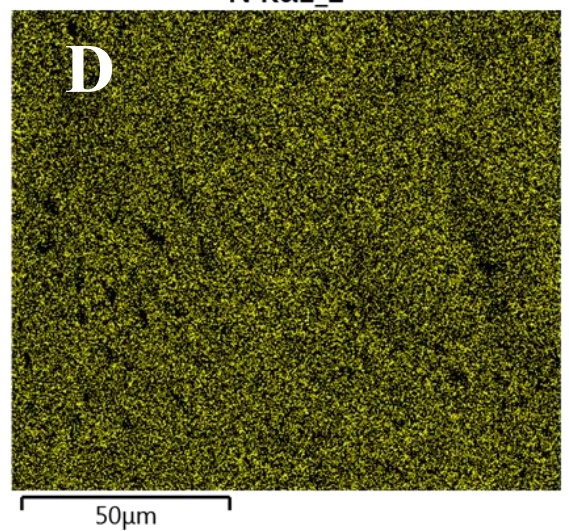

Figure 1. SEM images of the cross-section of LDPE melt-processed with $40 \mathrm{wt} \%$ DNA prepared via (A) freeze-fracture or (B) cryo-microtoming. EDS maps of the nitrogen signal, shown in yellow, collected at $10 \mathrm{kV}$ for the (C) freeze-fractured and (D) cryo-microtomed surfaces. The cryo-microtomed surface yielded more accurate information about the dispersion of DNA within the LDPE matrix. The SEM and EDS images were collected using a Nova Nanolab 200 coupled with an XMax $50 \mathrm{~mm}^{2}$ EDS detector. 